\title{
Index
}

abortion rights $52,71-2$

academic communities 9-10, 43-5, 74-6

discrimination in $37-8$

privilege in 22

activism 13,15

art-based 186

online 216

aesthetics 31-1, 56-7

Ahmed, Sara 21, 28

Ahmed, Taha 198, 200

Akomfrah, John 95

Algonquin people 133

Ali, Tariq 4

Alliance for Choice 52

'alt-right' movement 30

Amponsah, George 84

Amritsar 182, 185

Antonini, Francesca 213-14

Anzaldua, Gloria 25

apartheid 6

Arafat, Yasser 26

art

concept and definition of xix, 222

democratising access to 204-6

development in India 194-5 potential for creativity 208

'real' 8

as a socially-progressive tool 206

study of 195

world $145-6$

art collections 24-5

art exhibitions 59-60, 201, 216, 222

artist residency programmes 194-204,

207, 221-2

artistic expression $32-3$

social commentary in 27

artists

need for transformation in

treatment of xxi

support for and recognition of

$\mathrm{xx}$

assimilation policy 88

asylum seekers 30, 78, 176

Auguiste, Reece 97-8

Australia 3

authoritarianism xix, 32, 214

Auzaar (film) 196

Avaaz group 36

Bailey, David A. 88

Bajaj, Manvi 197 
Balibar, Renée 212

Banksy 25, 32

Bashir, Saiyna 199

Batalvi, Shiv 183

'bearable life' concept 21

Being Black (film) 81

Bell, David M. 43

belonging, sense of 46

Berger, John 135-8

Berlant, Lauren 21

Bethlehem 26

Bhattacharyya, Gargi 134

Bidisha ix, xvii-xxi

biennials 194

Binder, Toby 26

Black, Asian and minority ethnic

(BAME) groups 81-91, 101-10, 217-18

Black Britishness 37, 81-3, 86-94

Bloch, Ernst 46

'Bloody Sunday' (1972) 143

Boal, Augusto (and Boalian methodologies) 159-61

border art and cultures 25

borders and bordering 12, 38, 89-90, 195

Bourdieu, Pierre 213

Brazil 3

Brexit xix, 1, 4, 9, 26, 38, 42, 90, 103, 133

Brexit Party 131-2

BRIT Awards 27-8

British Broadcasting Corporation (BBC) xviii

British Empire 221

British Film Institute African Caribbean Film Unit 94 Creative Interruptions Festival 42

Brolly, Francie 219-20

Brown, Sean 153

Burjapati, Rachita 197

Burma 3
Burnett, Charles 97

Butalia, Urvashi 181-2

Cady, Stephen 159

Callanan, Saoirse 49-50

capitalism 27, 31, 120, 136

corporate 9

neoliberal 3

see also racial capitalism

Catney, Tony 49-50, 167, 172-5

Celada, Nina 197

Charlottesville incident (2017) 3

China 3-4

civil rights, historical and contemporary 41

civil rights movement $6,14,47-51,54$, $143-5,168,219-20$

Clancy, Sarah 30

class divisions 54-5, 130-1, 135

Cleary, Joe 9

co-creation of knowledge 10, 12, 15-16, 41-9, 52-6, 70-6, 85, $160-3,168,172-6,215-16$

paradox of 45

collaborative research $10-13,42-8$, $54-6,60-2,71-3,214$

possible failure of 44

colonialism and decolonisation 5-9, 13-16, 26-7, 33, 43, 68-74, 87-91, 98-101

'colour bar' 88

community, sense of 192

community involvement 11

Connor, Naomi 165-6

contemporary art 25

contractual workers 128

Coutinho, Agnieszka ix, 114, 118, 121-30, 133, 138, 218-19; co-author of Chapter 4

creative and cultural industries (CCIs) 82-7, 105-10, 217-18

Creative Interruptions Festival (June 2019) 42 
Creative Interruptions project 2, 11, $37-8,41-8,55-7,70-1,75,84,91$, $106,109,114-15,121,130-3,151-4$, 186,220

in Punjab 57-68

Creatively Connecting Civil Rights (CCCR) project 142-9, 153, 156, 159-61, 164, 173-5

creativity $\mathrm{xx}-\mathrm{xxi}, 1-2,6-9,26,29-36$, 46, 119-23, 187, 207, 211-14, 218-19, 223

everyday form of 119-22, 184, 186, 193,218

grassroots form of 7

criminality 31

Crow Nation 21

cultural expression 31, 33

Cultural Resource Conservation Initiative 15-16

cultural trends, contemporary 42 cultural workers 7-9, 82-3, 86, 93, 215,218

interaction with ordinary citizens $65-7$

Daniels, Jessie 30

Dash, Julie 97

decolonisation see colonialism

democratic values 217

deregulation 137

Devane, Tom 159

Dhariwal, Parvinder 191

Didi, Madan 185

Didi, Sheila 185

digital technology 216-17

discrimination 142

disenfranchised groups 45, 161, 214

Doyle, Roddy 211

Drill 31

Du Bois, W.E.B. 5

Dublin Pride parade 30

Duggal, Kartar Singh 189-91
Eagleton, Terry 148

Edensor, Tim 218

El-Tahri, Jihan 93

Emerson, Ralph Waldo 190

English language 73, 126

environmental crisis 193

epistemology 69

Esch, Elizabeth 134

Esfandiari, Mehrshad 49-50, 164-8, 173-4

ethnic minorities 89

see also Black, Asian and minority ethnic groups

Eurocentric ideology 69

European Union (EU) 36

citizens of 90

extremism 4-5

Facebook 4, 35

Faiz, Faiz Ahmed 8, 183

'fake news' 4

Farage, Nigel 131-2

Faris, Matt 147, 152-4, 161-2, 175

Fatima, Kanza 198, 200

Féile an Phobail 35

films and filmmaking 26, 33, 81-110, 216-20

Finlay, Tom 51, 53, 158

foreign workers 125-9, 135

Foucault, Michel 70

Fox, Hannah 161

Fox, Jonathan 161

Foxconn (company) 4

funding of research $74-5,151-2$

Gandhi, M.K. 183

Gaza 23

Gearing, Jay ix, 114-21, 132-3, 138, 218-19; co-author of Chapter 4

gentrification 48

Gerima, Haile 97-8

Gibson, Rachel K. 216

Gilmore, Ruth Wilson 136 
Gilroy, Paul 30

Giroux, Henry 213

Givanni, June 92-4, 98, 101

global financial crisis (2008) 3, 136

Gordon, Avery 70, 132-3

Gould, Gaylene 96-101

Gramsci, Antonio (and Gramscian thinking) $16,65,213-16$, 222-3

Green Revolution 192

Green Shoot Productions 51, 149-51, 158,163

Grenfell Tower fire (2017) 27-8, 103

Grime music 31

Grime4Corbyn $\left(\mathrm{G}_{4} \mathrm{C}\right)$ campaign (2017) 27

group dynamics 75

Grunwick 6

Gucci (fashion brand) 95

La Haine (film) 211

al-Haj-Ali, Mohammed 27

Hall, Stuart 72, 86-90, 135-8

happiness, concept of 21

Haraway, Donna 70

Hargey, Fionntán ix-x, 14-15, 51, 144-54, 166, 174, 219; co-author of Chapter 5

Hasan-Bounds, Daisy x, 42-3, 214, 216; co-author of Chapter 2

hate speech 4

Hawal, Kassem 34

Hawkins, Harriet 29, 119-20

Hirsch, Marianne 20

Holdman, Peggy 159

Holmes, Seth 136

Home Office 1

homecoming, sense of 46, 214

hooks, bell xviii, 12-13, 19-22, 42, 161, 217

hope for the future $21,213,221$

Horner, Lindsey K. 160 'hostile environment' policy 1-2, 6, 9, $38,89-90$

Housing for All 52

human relationships and human values $71-3$

human rights 146

Hungary 3

immigrants 122-4, 135-8

back-and-forth or migration for settlement 137

housing for $137-8$

India 3, 24, 38, 47, 57-68, 87

Indian People's Theatre Association (IPTA) 188

indigenous knowledge 70 inequality $27,32,183,214$ Institute of Race Relations 132 institutional racism 3, 217 international law 5, 23 internet resources 216 interruption of dominant regimes 32-9 Iosifidis, Petros 217 Islam, Asiya 9-10

Israel 5, 23-6, 33, 36, 38, 221

Jafa, Arthur 95

Jaganathan, Aditi x, 217; co-author of Chapter 3

'Jago', practice of 215-16

Jameson, Fredric 46

Jith, Athul 196-7

Johnson, Linton Kwesi 6

JR (artist) 25

Kane, Robb 49-50

Kassovitz, Mathieu 211

Kelly, Susie 50

Kenins, Laura 203

Kensington and Chelsea, Royal

Borough of $26-8$

Khan, Furrukh A. 199

knowledge exchange 15 
Labour Party 90, 132

labour shortages 87

Lahore 182

language 45, 189

see also English language; Punjabi

language

Lansiquot, Rabz 95, 101

Lear, Jonathan 21

Ledwith, Margaret 160

left-wing ideology 188

LGBTQ+ groups 47-51, 55, 146, 153, $156-7,170$

'lived theory' concept 11-13, 27, 43, 46, $70-2,75,132$

living research $72,75-6$

Lowe, Lisa 135

Lowkey 27-8, 31

Luchoomon, Krishna 200-1

Ludhianvi, Sahir 183

Lynch, Martin X-xi, 14-15, 49-55, 144-58, 161-2, 166, 169-75, 219; co-author of Chapter 5

Lyric Theatre, Belfast 14, 47, 49, 54-5

McCann, Eamonn 156

McDaid, Emer 50

McElhinney, Matthew 155, 167, 171, 175

McGee, Noel 50

McKeagney, Orla 51-3, 56, 154, 161-4, 172, 175

Maguire, Áine 49-50, 168-71, 175

Mahdi, Warsame 49-50, 162-4, 168-74

Mahn, Churnjeet xi, xvii-xviii, 57, 6o-3, 185, 215, 221-2; co-editor and co-author of Introduction, Conclusion and Chapters 1 and 6

Makeba, Miriam 6

Malik, Sarita xi, xvii-xviii, 6o, 63, 67-8, 214-17; co-editor and co-author of Introduction, Conclusion and Chapters 1, 2 and 3
Manto, Saadat Hasan 200

marginalised groups 47-8, 75, 215

marginality and marginalisation $\mathrm{xxi}$, $1-2,37,44-5,86,90,207$

Marin, Louis 46

Market community in Belfast 146-7

Marquez, Gabriel Garcia 211

Maula Jutt (film) 33

May, Theresa 27-8, 89

media, corporate 3

Meir, Golda 33

Mela festival 57-61, 64-8, 71, 201-8

democratising access to art 204-6

Melaugh, Eamon 156

memorialisation 24

Mercer, Kobena 89

'metaxis' 159

'micro-sociality' 56

migrant workers 3, 5, 113-14, 218

challenges faced by 1

militarisation 24

Mir, Farina 182, 189

Mohr, Jean 135-8

Moore, Ruth 150, 153, 158

Moten, Fred 98, 100

Mulhern, Francis 174, 212

Mullan, Seán 49-50, 168-71

multiculturalism 89

Muñoz, José Estaban 21

Murphy, Anne xi-xii, 185, 221;

co-author of Chapter 6

museums xxi

music 6-7, 31, 120-1, 206

nationalism 2-6, 72-4, 184

Nazi regime 3

Neelands, Jonothan 159

Nehru, Jawaharlal 183

Nelson, Bethany 159

neoliberalism 3-5, 31, 37, 94, 96, 114, $133,213-14$

Nkiru, Jenn 94-5, 101 
Northern Ireland 6, 9, 14, 24, 26, 41, 47-51, 71, 219

Northern Ireland Civil Rights

Association 143

Notting Hill Carnival 35

Oates, Sarah 216

Ó Dochartaigh, Fionnbarra 156

O’Dowd, Kellie 165

Onasanya, Fiona 131

optimism of the will 222-3

oral history $184-5$

'otherness' 3, 29, 69, 83

outreach 158

over-theorisation 46

Pahl, Kate 43

Pakistan 24, 33-4, 68, 87

Palestinians and their cultural resources $\mathrm{xx}, 5-6,23,25,33-4$, $38,220-1$

Pangur Bán Literary and Cultural Society 147

Parmar, Pratibha 100

participation in research $10-11$

Partition

of India 181-6, 195-203, 221

of Ireland 142, 186

of Palestine 186

Partition Museum, Amritsar 185

patriarchy 97

Peach, Blair 132

Peshawaria, Shashank 198-9

Peterborough 114-15, 131-3, 136, 186 the Philippines 3

Pierse, Michael xii, xvii-xviii, 15, 50-1, 54-6, 144, 149-53, 157-74, 219; co-editor and co-author of Introduction, Conclusion and Chapters 1 and 5

Playback Theatre 159-63, 174

Plenty Coups 21

podcasts 101, 105-8, 217-18
Poland 3

policing 89

'political Blackness' 6, 89

populism 29

Powell, Enoch 6

power relations in research $43-5,56$, $70-6,160,175,218$

precarity $72-3,193,217$

Preet Lari (literary magazine) 183-5, 190-3, 197

Preet Nagar 57-66, 73, 181-4, 190-207, 221-2

politics of collaboration in 60-5

Premchand, Munshi 190

Prescod, Colin 81-2

Pritam, Amrita 183, 190

Progressive Writers' Association 16, $183,186-8$

protest movements 7,36

Punjabi language 188, 190

Putuma, Koleka 212

queer politics 21

racial capitalism $134-5,138$

racialisation $1-5,10,13,29-31,82-3$, 90, $110,133-8$

racialisms 83

racism $2,6,12,30,38,54,69,71$, $88-91,130-1$

'radical openness' xviii, 12, 42, 223

in a hostile world 19-23

promise and reality of 44-7

radicalisation 91

Raghu KV 62-3, 67

Ramamurthy, Anandi 6, 110, 220

Rana Plaza collapse (2013) 4

Rancière, Jacques 35

Rao KV xii-xiii, 185, 200-1, 221; co-author of Chapter 6

refugees 3, 24, 73, 191, 195

reggae 6

resilience $2,19,23,34,35,120,219,221$ 
resistance to oppressive ideologies 29-32, 37, 186, 219-23

right-wing politics $2-6,30$

riots 88

Robinson, Cedric 134

Robinson, Imani 95

Roediger, David 134

Rogaly, Ben xiii, xvii-xix, 47, 114, 116, 119, 130-8, 218-19; co-editor and co-author of Introduction,

Conclusion and Chapters 1 and 4 Rolph-Trouillot, Michel 220-1

Rushdie, Salman 211

Saha, Anamik 85

Salas, Jo 161

Sansour, Leila 26

Scott, James C. 14

screen media 13, 83, 110

see also films and filmmaking

Sekhon, Sant Singh 191

self-awareness xviii

self-reflexivity 22

Shammalah, Fadi Abu 23

'Shoe' protest (Brussels, 2018) 36

Simpson, Lucy 50

Singh, Gurbakhsh 57, 183-4, 190-1, 196

Singh, Hridaypal 190

Singh, Jasber xiii, co-author of Chapter 2

Singh, Mohan 190

Singh, Nanak 190

Singh, Poonam xiii, 60, 185; co-author of Chapter 6

Singh, Rati Kant 185, 200

Singh, Ratika xiii-xiv, 59-66, 185, 197; co-author of Chapter 6

Singh, Samia xiv, 59-63, 66-8, 185, 197, 202; co-author of Chapter 6

Singh, Sumeet 185

Sivanandan, Ambalavaner 11, 43, 70, $74,88,132-3,216$ small-group play (SGP) processes $164,169-76$

Smith, David Norman 222

Smith, Tuhiwai 11

Sobieraj, Agnieszka 118

social housing 27

social justice xxi, 9, 83

social media $3-4,23,35,84,86,197$, 217

social research 161

socio-political agendas 32

solidarity 35, 38-9

Spivak, Gayatri C. 69

Srishti Institute of Art and Design 57-9, 184

Standing Rock 35

Stormzy 27-8, 31

Suleiman, Elia 26

Suresh, Sreshta 196

Szczepaniak, Joanna 116-17

Thatcher, Margaret 114

theatre-based research $\mathrm{xx}, 14-15,41$, $47-8,51-6,71-5,150,154-61$, 166-70, 174-5, 215, 219

The Specials 6

theory related to practice 11

Thoreau, Henry David 190

Tolstoy, Leo 8

totalitarianism 29

trade unions 124,130

Trump, Donald 25, 103

Turkey 3

\section{United Nations 23}

United States 3, 25

university-led research 43-4, 214

utopian texts $46-7,184$

Valluvan, Sivamohan 5, 90

vernacular creativity 184, 212, 218

see also creativity

Vettraino, Elinor 159 
Index

Vir Singh, Bhai 190

Virdee, Satnam 133

Virginia (US state) 133-4

Virk, Kulwant Singh 190

virtual reality (VR) 68

Vrikki, Photini xiv, 106, 107, 217;

Wad, Gavati 195-6

Wagah-Atari border 24

walls $24-7$

Web 2.0 tools 217

We'll Walk Hand in Hand (WWHIH)

(play) 49-50, 142-4, 148-9, 155-6, 166-75, 219-20

We Must Dissent (film) 147, 162

Wheeler, Mark 217

white supremacy xvii, 3, 5, 30, 69

Whitman, Walt 190

Wiegman, R. 30

Williams, Raymond 119
Windrush scandal 2, 87, 89 women, role and status of 69

Women's Resource Development Agency 52

Workers (film) 113-21, 129-30, 133, 136, 218-19

working-class experience 27

working practices 4, 113, 118-19, 122-31, 134-7, 219

Workplace (book) 137

xenophobia 88

Xie, Shaobo 221

YouTube 4, 102-3

Yuusuf, Maryama 40-50, 53, 168-71, 175

Zaman, Rehana 98-101

Zephaniah, Benjamin xiv-xvi 